\title{
El imaginario franciscano y el proceso de evangelización novohispana en el siglo XVI
}

The Franciscan imaginary and the New-Spanish EVANGELIZATION PROCESS IN THE XVI CENTURY

\section{Oscar Fernando López-Meraz*}

* Universidad Veracruzana, México Correo-e: sthi2009@live.com.mx (iD) orcid.org/0000-0002-1185-642 Recibido: 20 de marzo de 2019 Aprobado: 17 de marzo de 2020
Resumen: Se presenta un acercamiento a la categoría 'imaginario social' y se caracteriza a las crónicas religiosas, algunos elementos del espacio y seres fantásticos pertenecientes a la mentalidad occidental que aparecen en la descripción de lo americano en las obras de dos de los franciscanos más destacados del siglo XVI en Nueva España: Bernardino de Sahagún y Jerónimo de Mendieta. Se explica cómo estos religiosos evitaban la incomprensión del otro al sumar la cosmovisión y elementos mesoamericanos al plan providencial cristiano. El traslado del imaginario cristiano permitió evitar, al menos por un tiempo, la ruptura del mundo pensable e imaginable.

Palabras clave: historia social; América Latina; España; cultura europea; comunidad religiosa; forma y género literario

Abstract: An approach to the 'social imaginary' category and the characterization of religious chronicles, some elements of the environment and fantastic beings, which belong to the occidental mentality, that appear in the descriptions of the territories in the works of two of the most distinguished Franciscans in the XVI century in New Spain: Bernardino de Sahagún and Jerónimo de Mendieta. It is explained how these friars avoided the other's incomprehension by adding pre-Columbian worldview and elements to the Christian providential plan. The transposition of the Christian imaginary prevented, at least for a while, the rupture of the thinkable and imaginable world.

Keywords: social history; Latin America; Spain; European cultures; religious communities; literary forms and genres 
INTRODUCCIÓN

1492 es uno de los años más recordados en la historia de la humanidad. La llegada de los europeos a tierras americanas marcó un parteaguas para el futuro de los habitantes de los dos continentes, influenciando y afectando también a otros sujetos en Asia y África. La conquista del espacio continental inició en 1519, con la llegada de Hernán Cortés a litorales mayas, y se concretó, para el caso mesoamericano, en 1521, con la caída de México-Tenochtitlán. Por ello, desde este año hasta 2020 , tanto en la producción historiográfica profesional como en la opinión públi$\mathrm{ca}$, han abundado resultados de investigaciones y reflexiones sobre los procesos que llevaron a España a dominar gran parte de lo que hoy es Latinoamérica y otros territorios, como Filipinas.

El clásico concepto de 'descubrimiento' fue muy cuestionado, así como la mal llamada conmemoración del 'Día de la Raza', principalmente a finales de la década de los ochenta e inicios de los noventa del siglo pasado, cuando diferentes movimientos de pueblos originarios hicieron más visibles sus protestas por la violencia y exclusión de las que son blanco. Posteriormente, se fue posicionando como un sustituto el término 'Encuentro de Dos Mundos', promovido por Miguel León-Portilla.

Tanto la palabra 'descubrimiento' como 'encuentro' anulan toda la violencia ejercida por los occidentales en el continente americano. La primera da por hecho que América no existía antes de los españoles, lo que es completamente falso al haber civilizaciones tan avanzadas como los mexicas y los incas, sólo por citar los ejemplos más conocidos. La segunda encubre los hechos, pues un encuentro aparenta un trato casi amistoso entre los sujetos involucrados. No por nada Edmundo O'Gorman escribió el artículo "La falacia histórica de Miguel León Portilla sobre el «encuentro del Viejo y Nuevo Mundos»" (1987). Otra reacción a la propuesta del famoso nahuatlato fue la publicación de
1492. El encubrimiento del Otro. Hacia el origen del "mito de la Modernidad" (1994), de Enrique Dussel, donde el filósofo sostenía que el otro no fue descubierto, sino 'encubierto' como lo mismo.

Más propositivo al análisis resulta el concepto de 'invención' planteado por el mismo Edmundo O'Gorman (1977), aunque no fue él quien lo usó por primera vez, pues ya en la primera mitad del siglo XVI (más exactamente, en 1528) Fernán Pérez de Oliva había escrito una obra bajo el título Historia de la invención de las Yndias (1965). A diferencia del autor mexicano, el español comprendía invención como 'descubrir'. O'Gorman, por su parte, asentó que la idea de descubrimiento pertenecía al proceso de invención de Améri$\mathrm{ca},{ }^{1}$ negando que correspondiera a un proceso meramente físico (1977: 9). Así, 'inventar' América se volvió un punto de conexión entre una filosofía de la conciencia occidental y su expresión en el lenguaje.

En 2009, José Rabasa señaló que la noción o'gormaniana también presentaba serias limitantes al sólo oponer 'descubrimiento' e 'invención'. En su lugar, analizó una serie de autores que han hecho uso del concepto de 'invención', ampliando su abanico explicativo para comprender emergencias mitológicas, procesos culturales, formas de vida cotidiana y racismos. Entre quienes destacó están Roy Wagner (1981) y Michel de Certeau (1999). Aquí, sin embargo, pretendemos

1 Si bien aquí se mencionan algunos de los conceptos centrales del tema, no perdemos de vista que existen otros sobre los que merece reflexionarse más. Entre éstos destaca la misma categoría de 'conquista', que ha sido analizada a partir del ejercicio ya consolidado de quince años dirigido por Guy Rozat mediante el seminario "Repensar la Conquista". Por supuesto, no se puede olvidar la corriente historiográfica denominada 'Nueva historia de la Conquista', encabezada por Matthew Restall. En otro sentido, sobresalen las nociones de 'evangelización', 'aculturación', 'transculturación', 'hibridación' y 'sincretismo', como señala Norma Durán en "La evangelización de Mesoamérica en el siglo XVI: una aproximación crítica". En este artículo, la autora profundiza en el sincretismo, al que comprende como un elemento conceptual que ha trivializado la "complejidad del choque de ambos mundos, pues se asumen las diferencias, en el mejor caso, como resultado de esta fusión, lo cual implica que las sociedades prehispánicas rechazaron o mal asimilaron la nueva religión” (Durán, 2016: 117). 
abonar a la acción de invención o'gormaniana con clave de encubrimiento, pero colocando el foco de atención en una parcela del imaginario occidental comunicada por algunos de los más destacados conquistadores espirituales (por recuperar la noción de Ricard, 1986): frailes franciscanos. Dos son los ejes en los que se concentra este texto: espacio imaginario y seres fantásticos. Antes de ello, se identifica la noción de 'imaginario' que guía el trabajo.

\section{IMAGINARIO SOCIAL}

'Imaginario' es una noción utilizada con frecuencia, pero no siempre conceptualizada con rigor. Aquí, más que ensayar elementos nuevos para esta categoría, se identifican algunos esenciales para construir una interpretación sobre su presencia en las crónicas franciscanas del siglo XVI. Lo primero que hay que reconocer es que el imaginario social dirige y moldea la experiencia al someterla a lo ya conocido. En este sentido, funciona más como un esquema que permite percibir, explicar, intervenir y comunicar. Tanto escalas espaciales como elementos temporales, expresiones culturales, concepciones sobre lo humano y marcos racionales y emocionales (es decir, cómo se debe pensar y sentir) están enclavados en el imaginario social, pero también pertenecen a éste las formas de proceder, por ejemplo, por medio de tácticas o estrategias (Randazzo Eisemann, 2012).

Esto lleva a preguntarse si los imaginarios sociales construyen la realidad social. Sin duda, esto implicaría un largo y complejo debate, pero por lo pronto partamos de que son los sujetos históricos quienes elaboran, heredan y usan los imaginarios, en ocasiones de manera inconsciente o mecánica, para explicar el mundo e identificar el lugar que les corresponde (y el de los otros), así como las acciones que pueden y deben llevar a cabo. Al considerar los imaginarios como una masa de elementos que preconfigura la realidad, estamos muy cerca del pensamiento durkheiminano descrito en Las reglas del método sociológico (publicado por primera vez en 1895 y traducido al español en 1986) al comprender que las formas de pensar, actuar y sentir están cargadas siempre de una fuerza imperativa y coercitiva.

Una de las maneras más interesantes de abordar el imaginario es observarlo en coyunturas donde la experiencia se pone a prueba o se coloca en los límites, y en las cuales el conocimiento tiene posibilidades de aumentar. Sin duda, en esto cobra especial importancia el tema de la otredad, misma que es receptáculo de toda una serie de estereotipos y arquetipos. La función normativa del imaginario facilita la incorporación de espacios y sujetos ajenos a él, pues al menos para la época que aquí nos interesa no se podía participar o ser formado en dos imaginarios. Así, la mirada resultaba condicionada por un contexto histórico donde la observación era uno de los principales mecanismos del conocimiento.

Tenemos así que el imaginario social determina la interpretación sobre el otro. Sin embargo, el registro de las descripciones, o su profundidad, donde el imaginario traduce al otro, no existe en la misma proporción en los dos bandos confrontados (occidentales y 'naturales' para nuestro caso), o simplemente ha desaparecido para el caso de los 'vencidos'. Además de vincularse a un asunto de poder, el imaginario elabora y distribuye instrumentos para dirigir la percepción de lo social (Pintos, 2001). Así, se concentra en lo que considera importante, aquello que le permite mantener un orden y no romper esquemas.

De lo anterior se puede destacar que el imaginario social procura evitar la incomprensión a partir de la construcción de una serie de herramientas, como discursos, símbolos, conocimientos legitimados, actitudes y afectos. Todo ello permite la cohesión social e identidad entre los diferentes integrantes de un grupo, pues dichos elementos son difundidos mediante libros, canciones, pinturas, medios de comunicación e 
instituciones, como la escuela. No es de extrañar que los imaginarios sean construidos por los grupos hegemónicos y que ellos los transmitan al resto de la sociedad.

Uno de los representantes más reconocidos dentro de los estudios históricos de los imaginarios es el francés Jacques Le Goff (1995). Para este autor, quien propone un acercamiento diferente al tradicional (que se enfoca en lo 'empíricamente' demostrable y en las acciones de los 'grandes personajes'), el imaginario social se encuentra presente en los documentos y monumentos, con lo que responde a la crítica que se concentra en preguntar dónde está.

A partir de esta mirada, los imaginarios sociales pueden observarse, lo que constituye el principio fundamental de toda ciencia. Además, continúa el autor de El nacimiento del purgatorio (Le Goff, 1995), constituyen construcciones históricas y, por lo tanto, pueden ser reconocidos en los esquemas interpretativos de los grupos sociales contenidos en diferentes fuentes, como la literatura, el arte y algunas más donde es posible identificar el simbolismo articulador de lo cotidiano.

\section{LA NECESIDAD DEL Nuevo Mundo \\ Y LA IMPOSIBILIDAD DE SERLO}

Aun desconociendo la existencia de un 'nuevo mundo', Cristóbal Colón y sus compañeros de viaje/conquista llevaron a cabo todo un proceso de 'alterización' en las islas caribeñas. El asombro de un puñado de hombres, esperanzados en encontrar pronto tierra después de una travesía marítima anómala para la experiencia medieval, se fue convirtiendo en una práctica colonizadora que usó dos vías principales: la conquista armada y la espiritual.

La necesidad económica de una nueva ruta comercial hacia Medio y Lejano Oriente, debido al cierre del paso terrestre por los turcos otomanos, encontró en España a una de sus naciones impulsoras (la otra fue Portugal). La unión de Isabel y Fernando también le dio un cariz religioso a tal empresa, al considerarla punta de lanza del catolicismo. El mismo Colón era un sujeto profundamente religioso que consideraba la evangelización como uno de sus principales objetivos. La incapacidad de los conquistadores para reconocer que se encontraban en tierras inexploradas estuvo presente durante los primeros cuatro viajes que realizaron al continente.

La invención de América inició debido a ese desconocimiento; pronto se les nombró 'indios' a los naturales que vivían en las islas $\mathrm{y}$, después, a los que habitaban la masa continental. En el imaginario y la ambición occidental, India, Catay (China) y Cipango (Japón) eran los objetivos. Prácticamente desde el inicio de la empresa ibérica, los hoy llamados pueblos originarios sufrieron una muerte física y cultural. La espada, la cruz, las enfermedades y la esclavitud provocaron el deceso de millones de habitantes y la pérdida de elementos observables de su identidad, como templos y registros escritos.

Como explica José Rabasa (2009), la categoría 'Nuevo Mundo' no sólo debe ser comprendida como la representación del espacio imaginario producido por la idealización de paisajes deseados por Europa, sino como la presentación de la concepción moderna del mundo en tanto explotable. La exploración, además del conocimiento, buscó con frecuencia las mejores formas de dominio y saqueo. Pero ello no impidió que las descripciones de América se organizaran en clave metafórica y comparativa. Lo primero puede comprenderse porque el discurso no era plenamente científico, sino retórico, dirigido a convencer a los lectores (sobre todo a los poderosos, como los reyes), y no tanto a comprobar (finalidad del pensamiento científico moderno). Lo segundo se debe a que la escritura requería del pasado para darle nombre e identidad a la gente que habitaba en un espacio que ya era de por sí una completa sorpresa. 
Las Sagradas Escrituras, los autores grecolatinos, los padres de la Iglesia, las experiencias medievales y las que se presentaban en el momento fueron las fuentes de las que se alimentó la definición del otro. En mucha menor medida también se dio cabida a lo registrado por los sentidos, siendo la vista, como entre los griegos clásicos, el más importante. Pero si éstas fueron las fuentes de las que abrevaron los discursos, ¿cómo aparece lo americano en ellos? Aquí respondemos esta pregunta concentrándonos en las crónicas franciscanas. Observemos qué son, quiénes las escribieron y con qué fin.

\section{CRÓNICAS Y SU CONTENIDO}

La escritura de crónicas, que pertenecen a una corriente más amplia dentro del discurso occidental, encontró su auge en el siglo XVI. Herederas de la experiencia asiática, granadina, canaria y antillana, reforzaron el imaginario y construyeron justificaciones de la empresa colonizadora. Para el caso americano, su nacimiento puede fecharse en las postrimerías del siglo XV y dio sus últimos respiros con los jesuitas, cuya expulsión del continente se registró a finales del siglo XVIII. Es posible identificar dos momentos en este proceso, uno en donde los civiles se encargaron de la narración de los hechos con una mirada, en ocasiones, inquieta, sin recurrir en demasía al corpus descrito arriba; y otro en el que los religiosos se apoderaron de ella, utilizando toda la batería discursiva construida por Occidente a la hora de enfrentarse al otro.

Las crónicas constituyen testigos de debates historiográficos (por ejemplo, desde dónde debe escribirse sobre América, si desde lejos o en ella), espacios en los que se plantean las grandes discusiones de la época (métodos evangelizadores, características de lo humano), así como fuentes de primera mano para observar de qué forma se asimiló lo desconocido, entre otras muchas cosas. Como comenta Ignacio Arellano al referirse a las crónicas de Indias, existe "un mundo aún por descubrir" (2004: 9).

Los actuales análisis están abandonando el tradicional recurso historiográfico de usar las crónicas para decorar o corroborar algunas afirmaciones, no pocas veces con fines nacionalistas, como menciona Bustamante García (1990: 237), y están interesados en las relaciones entre verdad y ficción, los procesos de escritura y la construcción retórica de los personajes históricos, entre otros temas, pasando del estudio de las fuentes a la reflexión teórica que intenta analizar de qué manera se construyeron y comunicaron tales textos (Añón y Rodríguez, 2010). También existe una corriente que establece sistemas de interpretación de la historia y lo americano a partir de las concepciones de lo maravilloso, lo mágico y lo mítico.

La base para el acercamiento analítico a las crónicas y al resto de las fuentes coetáneas a ellas es rechazar la supuesta infantil credulidad de sus autores por registrar cosas y seres que, en nuestra mentalidad, podrían resultar irrisorios, además de observar con ojos críticos la forma en que están narradas victorias, derrotas, obstáculos, beneficios, sueños y esperanzas presentes en el 'nuevo' territorio.

De acuerdo con Antonio Rubial (2012), durante el periodo virreinal se pueden identificar cuatro etapas historiográficas en las crónicas religiosas. 1523-1560 es el periodo dorado de la evangelización y se caracterizó por el monopolio ejercido por el clero regular en la administración religiosa. Inició con fray Toribio de Benavente, Motolinía, y su objetivo principal fue informar a las autoridades sobre los avances del proceso evangelizador entre los nuevos hijos de Adán. Detrás de la escritura de este primer momento se puede identificar una filosofía de la historia que posicionaba a la Iglesia como la encargada de llevar a los fieles la salvación divina, y la intención de identificar las provincias como cuerpos sociales. La obra de Motolinía es la única conservada 
de esta época y en ella destaca la confrontación entre los mendicantes y los encomenderos por la defensa de los indios, pero también la justificación de la conquista.

Entre 1560 y 1650 la evangelización estaba avanzada, pero no consolidada; había voces de desilusión, como las de fray Bernardino de Sahagún y fray Jerónimo de Mendieta. También se presentaron acciones encaminadas a fortalecer la conversión religiosa y esfuerzos para regresar a la Iglesia primitiva practicada por la primera oleada de cristianos (Ruiz Bañuls, 2014). En otro sentido, se fueron sumando al registro las misiones desarrolladas en el norte novohispano y en Asia, siempre objeto de profundos intereses espirituales. Otro escenario nuevo lo conformó el entorno urbano, que además estaba integrado por una población pluriétnica. En este periodo se dio la aplicación de las medidas contrarreformistas y los concilios provinciales, así como el debilitamiento del poder regular y la participación de nuevos actores, pues a franciscanos, dominicos y agustinos se sumaron jesuitas, mercedarios, carmelitas y órdenes hospitalarias. Fue ésta una época de confrontación entre el clero regular y el secular, y entre peninsulares y criollos dentro de cada orden.

En la tercera etapa del ciclo de la crónica (1650-1750), los problemas internos pasaron a un segundo plano. Finalmente, el cuarto periodo (1750-1821) estuvo caracterizado por el apoyo total de la Corona al clero secular y, sobre todo, a las altas jerarquías episcopales peninsulares, lo que provocó un decaimiento en las órdenes religiosas que se reflejó en la historiografía. Aquí nos interesa mostrar cómo se manifestó el imaginario en el segundo momento, cuando al parecer recuperó fuerza, específicamente en lo relacionado con el espacio y los seres míticos.

Las imágenes y sus conceptos implícitos se apoyan, en ocasiones, en testimonios presenciales. En lo aquí presentado se pueden observar las relaciones entre memoria y percepción empírica articuladas por el imaginario occidental. Nuestra pretensión es acercarnos al estudio del imaginario vivo, recuperando la propuesta de Lefebvre (2006) sobre el pensamiento crítico interesado en estudiarlo. Para hacerlo, elegimos a dos franciscanos pilares de la evangelización novohispana en el siglo XVI: Bernardino de Sahagún y Jerónimo de Mendieta.

\section{IMAGINARIO Y ESPACIO AMERICANO}

Hablar de la imagen de la América del siglo XVI en el discurso conquistador requiere un acercamiento a Asia y al imaginario sobre ella. Casi desde el inicio del segundo milenio de la cristiandad, con el aumento de la población, una estabilidad económica mayor, el fin del miedo al año mil y el renacimiento de las ciudades, entre otros hechos, se produjo un mayor interés en este continente. Un caso representativo fue el de San Luis (Luis IX), monarca francés atraído por la idea de pactar con el gran kan para pelear con los creyentes de Mahoma, instalados en la península ibérica y presentes en el Medio Oriente, donde se ubica Jerusalén. La famosa ruta de la seda, que incluía también las especias, entre otros productos, resultó fundamental para la economía europea y la distinción de sus grupos sociales. La conversión de los pobladores de esos territorios motivó la visita de religiosos que, aún sin el éxito esperado, registraron espacios, hombres y otros seres.

A los nombres de los franciscanos Juan de Pian Carpino (1245), Guillermo de Rubrouk (1253), Odorico de Pordenone (1330), y el dominico Andrés de Longjumeau (1250) (Baudot, 2000), habría que sumarle el famoso Marco Polo (1298) y el menos conocido Niccolo dei Conti (1419). ${ }^{2}$ En los relatos de estos viajeros, la mirada occidental

2 Los años corresponden a la fecha en que estos personajes viajaron a Oriente. 
aplica categorías construidas para describir manifestaciones de lo lejano, por lo que los habitantes de Asia son calificados de exóticos, bárbaros y salvajes, términos en los que la misma noción de lo humano está en juego. Sin embargo, en dichas crónicas también existieron temas que fueron incorporados al imaginario para traducirlos al escenario cristiano. Así, por medio de mapas y otro tipo de registros, coexistieron lugares cuya existencia se había demostrado empíricamente, como Las Canarias o Madera, con otros pertenecientes a creencias antiguas, entre los que destacan las islas de San Brandán. Uno de los propósitos más importantes de los conquistadores fue localizar esos sitios en el Nuevo Mundo y otras latitudes, como África. El espacio americano fue acoplándose a los mitos occidentales. Uno de los ejemplos más destacados en este sentido fue el paraíso terrenal, del cual se creía que brotaban cuatro ríos distribuidos por el mundo.

Entre los esfuerzos por pensar este tema encontramos a Antonio de León Pinelo. Para este español, resultó evidente que en América del Sur se localizaba el paraíso, pues se ubicaba al oriente de la tierra adánica; además, reconoció en el maracuyá el fruto prohibido. De esta forma, no resulta extraño que el consejero real de España y cronista también situara en esa región del Nuevo Mundo elementos esenciales del mito cristiano, como el nacimiento del hombre y el Diluvio (De León Pinelo citado por Sérgio Buarque de Holanda, 1987: 18).

Sin ánimos de hacer una historia de la noción de paraíso terrenal, recuperemos algunas ideas básicas para después presentar cómo fue construida por los frailes franciscanos. En este mito existen nociones judeocristianas, cosmológicas griegas y, claro, medievales. Su ubicación geográfica fue cambiando: en un principio se pensó que estaba en una montaña cerca de la luna $\mathrm{y}$, posteriormente, con san Alberto Magno y santo Tomás, se creyó que debía estar más cerca de la Tierra, ya que: "no había de superar la primera zona del aire, cuya temperatura era la más apta para la constitución física del ser humano" (López Meraz, 2014: 25). En la transición del Medievo al Renacimiento se puede identificar la permanencia de esta imagen, que relaciona el paraíso con una montaña y la luna, tal como vemos en la obra de Dante Alighieri.

Prácticamente desde que la sorpresa de encontrar América se hizo presente entre los hombres del Viejo Continente, se desarrolló un ejercicio de coincidencia imaginaria. Varios de los exploradores más famosos afirmaron acercarse al jardín de las delicias, siendo, tal vez, el caso más significativo el de Américo Vespucio. Ya en la región continental, el franciscano del siglo XVI más famoso en nuestros días, fray Bernardino de Sahagún, afirmó que los mesoamericanos intentaron llegar al Edén:

venía[n] en demanda del Paraíso Terrenal,
y traían por apellido Tamoanchan, que quie-
re decir: «buscamos nuestra casa»; y poblaban
cerca de los más altos montes que hallaban.
En venir hacia el mediodía a buscar el Paraíso
Terrenal no erraban, porque opinión es de los
que escriben que está debajo de la línea equi-
noccial (2000: 20)

Según el fraile, los mexicas andaban en busca del paraíso terrenal, que se encontraba en la cumbre de una alta montaña, cerca de la luna. Para López Austin, dicha descripción parece concordar con una tradición indígena, según la cual Tamoanchan se ubica frente a volcanes nevados. Pero, añade el antropólogo, "ide dónde saca Sahagún que Tamoanchan estaba próxima a la luna?" (López Austin, citado en Contel, 2000: 620). Se trata de un símbolo inequívoco de la necesidad de evangelizar a los habitantes del lugar.

No fueron raras tampoco algunas comparaciones del medio físico entre el contexto americano y el europeo, como lo hace Motolinía con las heladas que se presentan en España y las de 
una localidad tan notable en fertilidad como ya lo adelanta su nombre: el Val de Dios. Es claro, nos encontramos frente a un sitio bautizado por la lógica cristiana.

La conquista del espacio, que se consideraba ya cristiano, permite observar las bondades tanto de derrotados como de aliados en el enfrentamiento entre españoles y mexicas. De este modo, la Ciudad de México era presentada con muchos esplendores, mientras que la provincia de Tlaxcala tenía "grandor" (García Icazbalceta, 1980) y en ella existían cosas notables, según anotó Motolinía. De esta forma se observa cómo el pensamiento de Sahagún, al que una tradición historiográfica ha calificado como padre de la antropología mexicana, participó en la descripción de la cosmogonía mesoamericana.

Hay otro espacio imaginario que resultó no sólo un referente para la acción religiosa novohispana, sino estructurante entre varios franciscanos. Al interior de la orden mendicante, Asia siempre fue un objetivo importante, no sólo porque a ese continente habían llegado los frailes menores en su primera misión fuera de Europa, sino porque se construyó un ideal acerca de sus habitantes. Asia se volvió un referente de añoranza. China atrajo a fray Martín de Valencia y a fray Domingo de Betanzos. Su intento de evangelización fue inspirado por la experiencia asiática de los frailes del siglo XIII, que consideraron a los habitantes de esa región los más hábiles para recibir la fe cristiana. La 'necesidad' que de Asia tenían los franciscanos se extendió hasta el periodo de 1808 a 1813 (ien plena guerra de independencia de México!), como lo demuestra Mariano López y Pimental, descalzo de la provincia de San Diego de México, quien presentó al gobierno español un plan para las misiones en México, China y Japón (Morales, 2008).

\section{IMAGINARIO Y SERES FANTÁSTICOS}

La mentalidad occidental creó un imaginario poblado de seres fantásticos. En América se dio continuidad al ejercicio desarrollado en otras coordenadas espaciales, como la India o Etiopía, donde se registraron especies monstruosas, gigantes, y un largo etcétera. Varios de los escritores más importantes ubicados entre finales del siglo XV y la siguiente centuria, entre los que destacan Sebastian Münster y Conrad Lycosthenes, son muestra de ello.

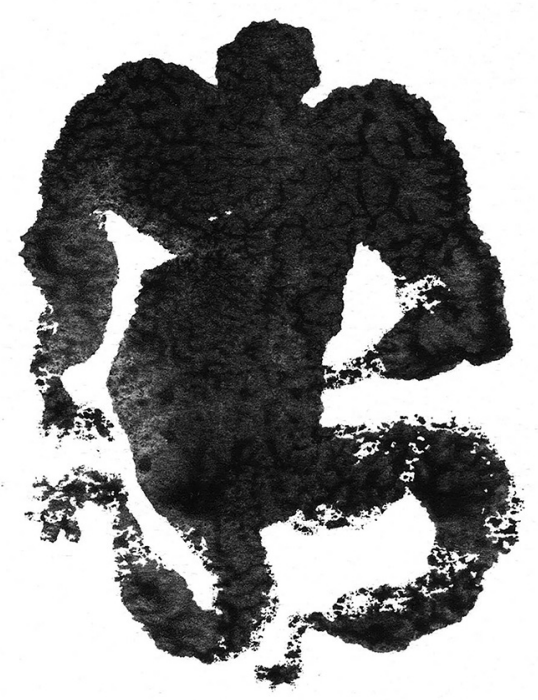

Huella de hombre, de la serie Obra pictórica (2018). Acrílico sobre papel: Carlos Alberto Badillo-Cruz. Prohibida su reproducción en obras derivadas. 
Cristóbal Colón, Pedro Mártir de Anglería y muchos de quienes pisaron de manera temprana las Indias Occidentales dieron fe de la existencia de sirenas, serpientes marinas, grifos, cinocéfalos, cíclopes, hombres con rabo y tritones. Tal como sucedió en el primer milenio de la cristiandad, los seres mitológicos cedieron terreno ante la presencia de manifestaciones demoníacas.

Hay, sin embargo, varios elementos aún presentes en crónicas franciscanas que permiten identificar algunos actores fantásticos. Así lo demuestra fray Bernardino de Sahagún al señalar la presencia del mazamiztli (que suma características de león y ciervo) a la manera del bestiario medieval. Estos animales fueron asociados con importantes valores simbólicos para la cristiandad, y representaron tanto a la figura de Cristo y al alma cristina, como a Satán y la herejía (Charbonneau-Lassay, 1997), una combinación perfecta para dar cuenta del mundo en el que vivían los naturales, cegados por la adoración al diablo, quien había promovido los más peligrosos pecados.

Más cercanas al imaginario cristiano occidental fueron las representaciones de serpientes con características femeninas. Fray Jerónimo de Mendieta identificó que algunos ídolos eran "como culebras y de éstas de muchas maneras, largas y enroscadas, y algunas con rostro de mujer, como pintan la que tentó a nuestra madre Eva" (Mendieta, citado en González, 1996: 100). Esto no resulta menor, pues la serpiente en la demonología ocupa una posición central como representación del mal. La razón es bien conocida: Satán adoptó su forma para seducir a Eva (Gen. 3:1).

Esto parecería un lugar común, pero Mendieta dio un paso adelante y se esforzó por reflexionar sobre la forma adoptada por Lucifer cuando provocó el pecado original. Resulta interesante que el mendicante no dudara sobre esto, pues todavía en el siglo XVI existía un debate sobre la presencia del diablo en ese momento crucial para la historia providencialista. El fraile lo tenía claro: se trataba de una sierpe con rostro de mujer (1971).
Según Jean de Cuba, el nombre para este tipo de seres era draconcópedas, de los que se sabía eran muy grandes y con caras de vírgenes humanas (Charbonneau-Lassay, 1997: 772). No es un dato sin importancia que los naturales tuvieran conocimiento, aunque éste no fuera muy claro, de la relación entre el diablo y Eva. Además, se seguía pensando en el paraíso como un escenario propio de América.

Sin duda, la producción simbólica relacionada con el diablo fue la más desarrollada por el imaginario cristiano en las conquistas americanas. ${ }^{3}$ Sahagún relacionó a Tezcatlipoca con Satán en el primer libro de su Historia general de las cosas de Nueva España, mientras que Mendieta lo conectó con el dragón, el único ser fantástico con valor negativo presente en la Historia eclesiástica indiana. Pero el imaginario de esta época también estuvo poblado por criaturas con una larga trayectoria en la mentalidad occidental.

Sin duda, entre los seres que aparecen con más frecuencia en las descripciones de los europeos sobre América se encuentran los gigantes. $\mathrm{Su}$ imagen fue relacionada con dos categorías básicas: barbarie/salvajismo, y primitivismo. Por supuesto, esto estaba directamente conectado con la supuesta superioridad occidental que impondría orden y civilidad.

En este sentido, se comprende perfectamente la descripción del padre Antonio Trello, quien afirmaba haber visto veintisiete gigantes con tres mujeres, e insistía en su dieta diaria: cuatro niños asados y una fanega de maíz (López Meraz, 2014: 36). Comunicar esta existencia se ubica en el corazón de un discurso que relacionaba al gigante con la barbarie, en donde la desmesura, así como su pertenencia y cercanía a la naturaleza resultaban características. Por supuesto, la presencia de tal primitivismo justificaba la acción

3 No es objetivo del presente trabajo ahondar en las representaciones presentes en el imaginario cristiano-occidental relativas al demonio en el siglo XVI novohispano. Al respecto, se puede consultar el texto "El Diablo en el discurso de evangelización novohispana del siglo XVI", de Berenice Alcántara Rojas (2013). 
occidental para sustituirlo por un orden civilizatorio, basado en lo moral y lo político. La instalación de la cristiandad permitiría una reducción de la bestialidad del gigante. Al respecto, Mendieta habló de 'medio gigantes', que a sus ojos ya no eran tan desagradables:

Y medio gigantes en nuestro tiempo los ha habido, uno en el pueblo de Cuernavaca, que tenía tres varas de medir menos una cuarta en alto, que son once palmos o cuartas de vara. Otro mozo hubo en Tecalli, y pienso que más alto, aunque más delgado de cuerpo, porque el primero era bien fornido y proporcionado (1945: 208).

El discurso de Jerónimo de Mendieta se nutre de tres fuentes: el pensamiento que pasa como propio de los naturales; el testimonio del franciscano fray Andrés de Olmos cuando visitó el palacio del virrey Luis de Velasco, el Viejo, en donde observó 'huesos de gigante'; y la propia memoria occidental de la que es heredero, con la que se constituyó como pionero de la etnografía mexicana (Ruiz Bañuls, 2010) y pasó a ser la "fuente de donde todos los arroyos que de esta materia han tratado, emanaban" (Mendieta, 1945: 82).

Es necesario notar que en los primeros escritos franciscanos el discurso cristiano también identificó al demonio con un gigante. Así lo hizo Olmos en su Tratado de hechicerías y sortilegios (1990: 43), cuando afirmó que en Tezcatépec el diablo se le presentó a algunos señores en esta forma. El núcleo del relato de fray Jerónimo de Mendieta es la solicitud del demonio para que los naturales mataran al guardián español Juan Cordero. Ellos decidieron, sin embargo, no ejecutar el mandato, sino comunicarle al europeo lo ocurrido. La reacción de éste fue enfrentar al gigante basado en la fuerza de la espada, sin recurrir al signo de la cruz y al nombre de Jesús.

La contienda reducida por el guardián al enfrentamiento de la carne y el hueso provocó que saliera malherido después de que su enemigo sólo usara un brazo. La representación del gigante en Olmos conserva mucha de la potencia violenta que el mundo medieval depositó sobre ella, pero su relato se construye desde la mirada de los primeros pasos de la evangelización; en cambio, Mendieta presenta una relación simbólica menos dura, da fe de la existencia de 'medio gigantes' y les otorga referentes más humanos que bestiales.

\section{ConCLusión}

La conquista de América representó un desafío para la comprensión occidental. La epistemología europea necesitó incorporar a los pobladores de este nuevo espacio en sus parámetros históricos e imaginarios. La experiencia se vio frecuentemente supeditada a la tradición. Viejas explicaciones fueron actualizadas y acomodadas a la observación de una realidad que no pudo ser reconocida como diferente.

Varios hilos conductores estuvieron presentes en el discurso de los primeros cronistas. Uno de ellos fue la definición de la naturaleza de los habitantes americanos; la polémica giraba alrededor de si eran animales o seres humanos. Conforme avanzaba el tiempo, los naturales quedaron inscritos en el discurso como hijos de Adán, pero menores de edad. Otro tema fue la imposición del imaginario occidental, directriz del pensamiento y la acción. De esta manera, se evitaría la 'incomprensión' por medio de un discurso compartido articulado por símbolos y conocimientos, entre otros elementos.

Los frailes franciscanos, representantes del grupo hegemónico interesado en la evangelización de los mesoamericanos, observaron en la cosmogonía mexica la búsqueda del paraíso terrenal en su afán por incorporarlos a su visión providencialista. Este concepto no pertenecía, por supuesto, sólo a los mendicantes, sino que era fruto de un ejercicio colectivo de la mentalidad occidental a la que pertenecían. Características 
como la cercanía de Tamoanchan a la luna no parecen explicarse de otra manera. La percepción de seres híbridos, como el mazamiztli, es muy probable que tuviera cabida desde el marco interpretativo del bestiario medieval.

Por su parte, en los relatos de los hijos de Asís se demuestra con claridad la necesidad de presentar el Nuevo Mundo como maravilloso. En los ejemplos aquí mencionados se observa cómo el espacio americano fue el depositario de una tradición que se remonta hasta el pecado original, con la transformación del diablo en serpiente con rostro de mujer, tema muy debatido en la mentalidad medieval y que Mendieta incluyó entre las creencias de los vencidos. Este mismo fraile incorporó en su obra el registro de gigantes, que estaban entre los seres fantásticos más importantes del imaginario occidental y representaban la barbarie y el salvajismo. Esto justificó la conquista espiritual, pues se consideraba a la cristiandad como el elemento central de la civilización.

La escritura de las crónicas se alimentó de elementos retóricos que incorporaron supuestos basados en la experiencia. Sin embargo, la memoria y toda una larga tradición a la hora de describir al otro guiaron la comunicación del espacio y el tiempo no cristiano, y a la vez facilitaron la incorporación de lo nuevo a lo viejo, a lo conocido. No era para menos: se intentaba evitar la ruptura del mundo pensable e imaginable.

\section{REFERENCIAS}

Alcántara Rojas, Berenice (2013), "El Diablo en el discurso de evangelización novohispana del siglo XVI", en Luis Millones y Alfredo López Austin (eds.), Cuernos y colas. Reflexiones en tomo al Demonio en los Andes y Mesoamérica, Lima, Fondo Editorial de la Asamblea Nacional de Rectores, pp. 101-127.

Añón, Laura y Jimena Rodríguez (2010), “¿Crónicas, historias, relatos de viaje? Acerca de los nuevos estudios coloniales hispanoamericanos", en VII Congreso Internacional Orbis Tertius de Teoría y Crítica Literaria, 18, 19 y 20 de mayo de 2009. Memoria académica, La Plata, Universidad Nacional de La Plata.
Arellano, Ignacio y Fermín del Pino (eds.) (2004), Lecturas y ediciones de crónicas de Indias. Una propuesta interdisciplinar. Actas del Quinto Congreso Internacional de edición y anotación de textos, Madrid / Pamplona, Vervuert / Iberoamericana / Universidad de Navarra.

Baudot, Georges (2000), "Los precursores franciscanos de Sahagún del siglo XIII al siglo XVI en Asia y América”, en Jesús Paniagua Pérez y María Isabel Viforcos Marinas (coords.), Fray Bernardino de Sahagún y su tiempo, León, España, Universidad de León / Instituto Leonés de Cultura, pp. 361-372.

Buarque de Holanda, Sérgio (1987), Visión del paraíso: motivos edénicos en el descubrimiento y colonización del Brasil, Caracas, Biblioteca Ayacucho.

Bustamante García, Jesús (1990), Fray Bernardino de Sahagún. Una revisión crítica de los manuscritos y de su proceso de composición, México, UNAM / Biblioteca Nacional / Hemeroteca Nacional.

Charbonneau-Lassay, Louis (1997), El bestiario de Cristo, Barcelona, Sophia Perennis.

Contel, José (2000), "Visiones paradisíacas: extrañas analogías entre Tlallocan y 'Paraíso Terrenal' en la Historia general de las cosas de Nueva España de fray Bernardino de Sahagún", en Jesús Paniagua Pérez y María Isabel Viforcos Marinas (coords.), Fray Bernardino de Sahagún y su tiempo, León, España, Universidad de León / Instituto Leonés de Cultura, pp. 617-630.

De Certeau, Michel (1979), La invención de lo cotidiano, México, Universidad Iberoamericana.

Durán, Norma (2016) "La evangelización de Mesoamérica en el siglo XVI: una aproximación crítica”, Historia y Grafía, año 24, núm. 47, pp. 115-143.

Durkheim, Emile (1986), Las reglas del método sociológico, México, FCE.

Dussel, Enrique (1994), 1492. El encubrimiento del Otro. Hacia el origen del "mito de la Modernidad", La Paz, Bolivia, Plural editores / Centro de Información para el Desarrollo.

García Icazbalceta, Joaquín (1980), Colección de documentos por la Historia de Mexico, t. I, México, Porrúa.

González, Luis (1996), Jerónimo de Mendieta: vida, pasión y mensaje de un indigenista apocalíptico, Mexico, El Colegio de Michoacán.

Le Goff, Jacques (1985), El nacimiento del purgatorio, Madrid, Taurus.

Le Goff, Jacques (1995), Pensar la historia, Barcelona, Altaya.

Lefebvre, Henri (2006), La presencia y la ausencia. Contribución a la teoría de las representaciones, México, FCE.

López Meraz, Oscar Fernando (2014), "Imaginario franciscano en Nueva España, siglo XVI: Demonio, paraíso terrenal, seres fantásticos y sucesos maravillosos", Amerika. Mémoires, identités, territoires, núm. 11, pp. 1-17, disponible en https://journals.openedition.org/amerika/6353

Mendieta, Fray Jerónimo de (1945), Historia eclesiástica indiana, Mexico, Editorial Salvador Chavez Hayhoe.

Morales, Francisco (2008), "De la utopía a la locura. El Asia en la mente de los franciscanos de Nueva España: del siglo XVI al XIX", en Elisabetta Corsi (coord.), Órdenes religiosas entre América y Asia. Ideas para una historia misionera de los espacios coloniales, México, El Colegio de México. 
O’Gorman, Edmundo (1977), La invención de América, México, FCE.

O’Gorman, Edmundo (1987), "La falacia histórica de Miguel León Portilla sobre el «encuentro del Viejo y Nuevo Mundos»", Quinto Centenario, núm. 12, pp. 17-32.

Olmos, Fray Andrés de (1990), Tratado de hechicerías y sortilegios, México, UNAM.

Pérez de Oliva, Fernán (1965), Historia de la invención de las Yndias, Bogotá, Instituto Caro y Cuervo.

Pintos, Juan Luis (2001), "Construyendo realidad(es): los Imaginarios Sociales", Realidad. Revista del Cono Sur de Psicología Social y Política, núm. 1, pp. 7-24

Rabasa, José (2009), De la invención de América. La historiografía española y la formación del eurocentrismo, México, Universidad Iberoamericana.

Randazzo Eisemann, Francesca (2012), "Los imaginarios sociales como herramienta", Imagonautas, vol. 2, núm. 2, pp. 77-96.

Rubial, Antonio (2012), "La historia eclesiástica en Nueva España”, en Rosa Camelo y Patricia Escandón (coords.), Historiografía mexicana. La creación de una imagen propia. La tradición española, Tomo 2, Historiografía eclesiástica, México, UNAM.

Ruiz Bañuls, Mónica (2010), "El discurso indígena en el proyecto evangelizador novohispano del siglo XVI", Revista Iberoamericana de Teología, vol. 6, núm. 11, pp. 9-30.

Ruiz Bañuls, Mónica (2014), "El franciscanismo en el contexto evangelizador novohispano: raíces del mensaje misional”, Sémata. Ciencias Sociais e Humanidades, vol. 26, pp. 491 507.

Sahagún, Bernardino de (2000), Historia general de las cosas de la Nueva España, México, Porrúa.

Wagner, Roy (1981), The Invention of Culture, Chicago, University of Chicago Press.
Oscar Fernando López Meraz. Doctor en Historia y Estudios Regionales por el Instituto de Investigaciones Histórico-Sociales de la Universidad Veracruzana (UV), México. Posdoctorante en la Universidad de Zaragoza y profesor de la UV. Sus líneas de investigación y generación del conocimiento son: historia de las mentalidades, representaciones e imaginarios del siglo XVIXVIII e investigación de didáctica y enseñanza de la historia. Entre sus publicaciones recientes se encuentran: "Conocer y representar al otro en América, siglo XVI. La actualización del pasado en el Nuevo Mundo" (Acta Classica Universitatis Scientiarum Debreceniensis, núm. 53); y "Experiencia de investigación sobre el proceso de aprendizaje-enseñanza de la historia en normalistas veracruzanos" (en Investigación Educativa en las Escuelas Normales, 2018). 